\title{
An incidental diagnosis...
}

\author{
I Khan, P Lambert
}

Musgrove Park Hospital, Taunton

\section{Introduction}

MEN-2a is a rare genetic endocrine disorder associated with mutation of RET oncogene on chromosome 10. It involves lesions in thyroid, parathyroid \& adrenal glands. Medullary thyroid carcinoma is the pathological hallmark \& usually the first presentation of this rare syndrome. Demonstrating RET mutation is of paramount importance for both patients \& their families.

\section{CASE HISTORY}

A 64 year old lady with IHD \&

hypercholesterolemia was referred to Endocrine clinic after she was incidentally found to have high Calcium (3.06) \& inappropriately high PTH (18.4) confirming the diagnosis of primary

hyperparathyroidism. The lady did not have a previous history of any endocrine problems \& her BP was well controlled with Atenolol \& Lisinopril. Fortunately the ultrasound neck \& SISTAMIBI scan were both concordant pointing towards a right lower parathyroid adenoma \& she was referred to ENT surgeon for minimal invasive parathyroidectomy.

Intraoperatively, the surgeon noticed a

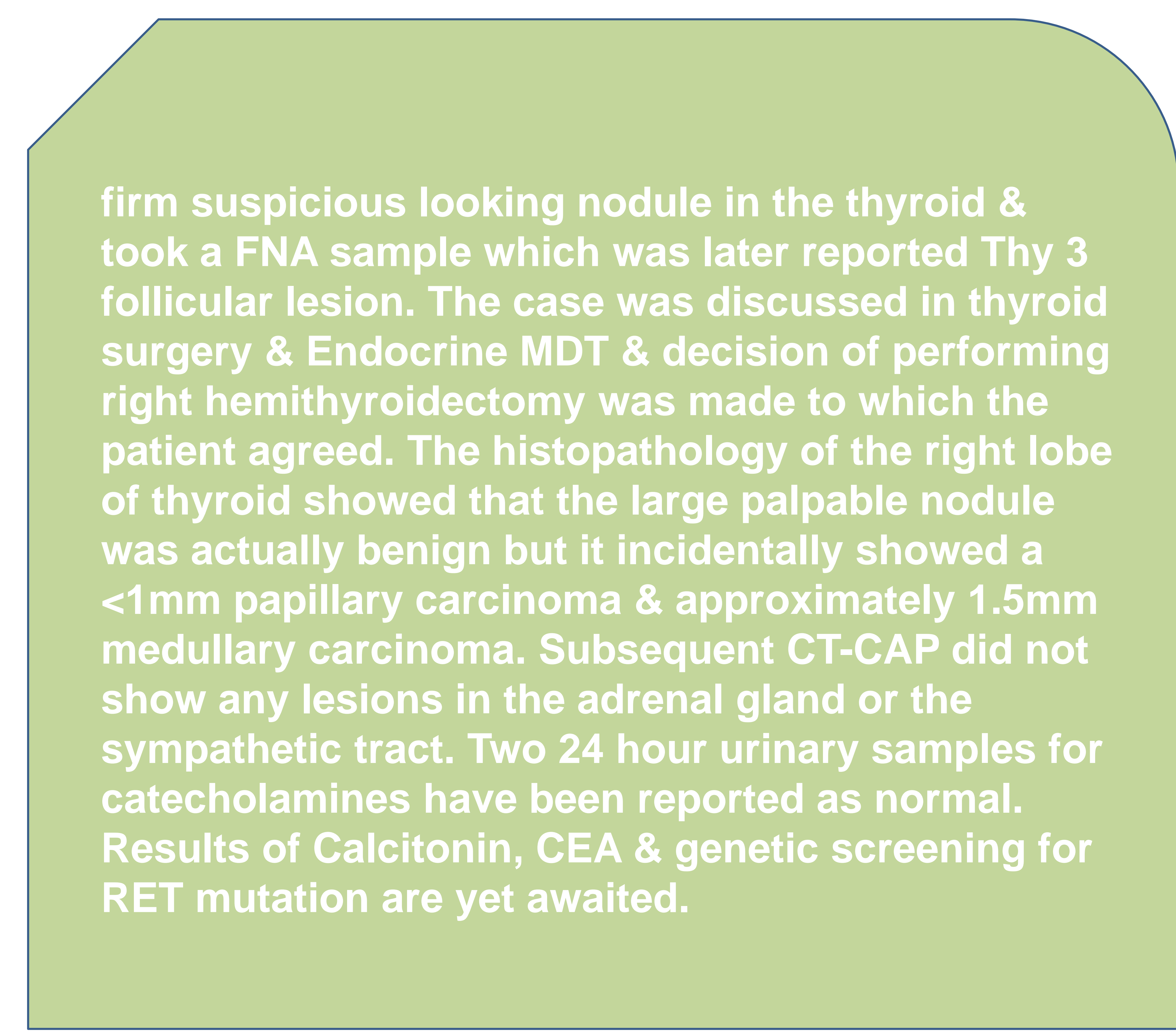

Clinical Features \& Manifestations of MEN 2a The presence of medullary thyroid cancer \& primary hyperparathyroidism raised the possibility of MEN 2a
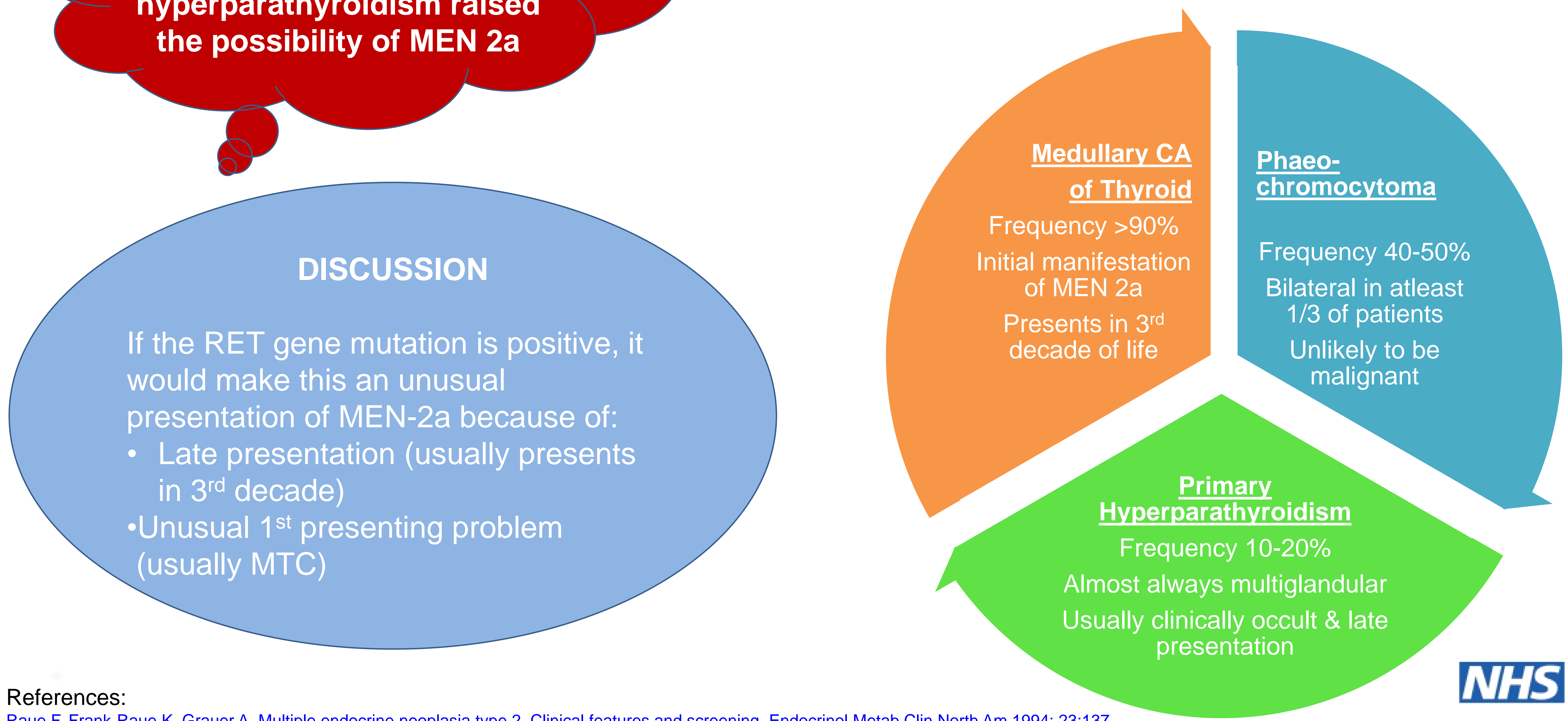